\title{
EFFECT OF HOME CARE WHITENING METHODS ON SURFACE ROUGHNESS OF LOW SHRINKAGE COMPOSITE (IN VITRO STUDY)
}

\author{
Mohammed A Elsayed*, Sameh M Nabieh" ${ }^{* *}$, Nady Mohammed ${ }^{* * *}$ and Muhammad AMasoud ${ }^{* * * *}$
}

\begin{abstract}
Purpose: The aim of this study was to evaluate the effect of home care whitening methods on surface roughness of low shrinkage composite. Methods: One hundred and twenty discs of low shrinkage (Filtek P90) were fabricated. Discs were divided into four groups according to the whitening agents (5\% hydrogen peroxide, sodium bicarbonate and whitening toothpaste) with control group brushed with distilled water only. Then the discs subdivided into three groups according to storage time in distilled water to (one day, three months and six months). The whitening agents were applied two minute per day by toothbrush stimulator apparatus. Ra values were determined by digital microscope with software program. Statistical analysis was performed by using ANOVA and Tukey's test. Results: The home care whitening agents significantly increased the surface roughness of low shrinkage composite, when the storage in distilled water caused decreasing of the surface roughness of low shrinkage composite. Conclusions: The home care whitening agents and the storage in distilled water had a significant effect on the surface roughness of low shrinkage composite.
\end{abstract}

\section{INTRODUCTION}

Esthetic dentistry has received increased attention in recent years. The demand for tooth whitening has increased dramatically over the past years with the result of development of many new whitening products from many different companies (1). A number of methods are available to improve the color of the teeth such as whitening toothpaste, professional stain removal, enamel microabrasion, vital tooth bleaching, non-vital tooth bleaching, crowns and veneers. Currently, gels, rinses, gums, dentifrices, whitening strips or paint-on films with low levels of carbamide or hydrogen peroxide are widely available ${ }^{(2)}$.

The brushing with toothpaste is the main method of oral hygiene, bringing many benefits, in addition to reduction in the incidence of caries. However, studies have shown that the movement of agents associated with the tooth brushing abrasive in a dentifrice and the toothbrush bristles, can cause damage to the brushed substrate and capable of altering the restorative material roughness ${ }^{(3)}$. Brushing with dentifrices is an example of a triblebody abrasion process, in which disaggregated particles slide between the tooth and brush bristles, the size of the abrasive particles and pressure being important factors in roughness and the speed at which the surface undergoes abrasion ${ }^{(4)}$. One of the factors that determine the clinical longevity of a restoration is its surface characteristics. Ideally a restoration must provide a smooth and regular surface, but it is not always possible, as the resin composites are frequently subject to certain deleterious actions in the oral cavity through the processes of abrasion, attrition and erosion ${ }^{(5)}$. The type of composite material is especially important from a clinical standpoint. Silorane-based composite was obtained from the reaction of oxirane and siloxane molecules. It has been claimed that this resin combines the two

\footnotetext{
* Dentist, Ministry of Health.

** Professor and Head of Operative Dentistry Department, Faculty of Dental Medicine, Al-Azhar University (Boys), Cairo. *** Assistant Professor of Operative Dentistry, Faculty of Dental Medicine, Al-Azhar University (Boys), Cairo. **** Assistant Professor of Dental Materials, Faculty of Dental Medicine, Al-Azhar University (Boys), Cairo.
} 
key advantages of the individual components: low polymerization shrinkage due to the ring-opening oxirane monomer and increased hydrophobicity due to the presence of siloxane ${ }^{(6)}$.

\section{MATERIALS AND METHODS}

The materials used in this study were:

1- Low shrinkage composite (Silorane composite).

2- Different three home care whitening agents (5\% hydrogen peroxide, sodium bicarbonate and whitening toothpaste).

\section{Specimen preparation}

One hundred and twenty discs were prepared by using specially designed custom made split circular Teflon mold ( $2 \mathrm{~mm}$ height and $5 \mathrm{~mm}$ diameter) enclosed within metal ring. The mold was placed on a transparent matrix strip supported by a glass slab then the mold was filled as one increment. Then the mold pressed by load pressure upon it by 200 gram weight to remove voids ${ }^{(7)}$. Then the glass slab was removed and the resin composite was light cured. The specimens were finished and polished.

\section{Grouping of the specimens}

The specimens were divided into four groups equally ( $n=30)$ according to type of home care whitening agents:

Group 1: immersion in hydrogen peroxide solution $5 \%$.

Group 2: brushing with wet sodium bicarbonate powder.

Group 3: brushing with whitening dentifrice Aquafresh intense white.

Group 4: brushing with distilled water as a control group.

Each group was subdivided into three equal subgroups $(\mathrm{n}=10)$ according to the storage period (one day, 3 months and 6 months).

\section{Application of the whitening agents}

The home care whitening agents were applied on the specimens for one minute twice daily. It was done either as (a) Immersion as in hydrogen peroxide solution or (b) By brushing as in sodium bicarbonate powder, whitening tooth paste or distilled water (The control group).

\section{Simulated toothbrushing procedure}

The specimens were put in a toothbrush simulator apparatus which consisted of battery powered electric brush which mounted to a fixed plate and the brush head touch a metal ring which hold the composite disc ${ }^{(8)}$. Load of the toothbrush standardized at $250 \mathrm{~g}$, soft toothbrush head with rotation movement and the head changed every three months.

The sodium bicarbonate and whitening paste slurry applied on the specimens as injected $2 \mathrm{ml}$ by a syringe every 10 seconds to keep the material cover the specimens.

\section{Storage}

All specimens were stored in distilled water media for storage periods one day, three months and six months and roughness measurement done at the end of every storage period. The specimens were stored in an incubator at a temperature of $37^{\circ} \mathrm{C}$ with $100 \%$ humidity.

\section{Surface roughness test}

The specimens were photographed using USB Digital microscope with a built-in camera (Scope Capture Digital Microscope, Guangdong, China) connected with an IBM compatible personal computer using a fixed magnification of 90X. The images were analyzed using WSxM software (5 develop 4.1, Nanotec, Electronica, SL). ${ }^{(9)}$ 3D image of the surface profile of the specimens was created. WSxM software was used to calculate average surface roughness $(\mathrm{Ra})^{(10)}$. The roughness was measured four times as a base line roughness, after twenty four hours, after three months and six months. 


\section{Statistical analysis}

Data analysis was performed by two-factors analysis of variance ANOVA test. One way ANOVA followed by pair-wise Tukey's post-hoc tests were performed to detect significance interaction between subgroups. $\mathrm{P}$ values $\leq 0.05$ were considered to be statistically significant in all tests.

\section{RESULTS}

\section{Effect of whitening agent groups on surface roughness}

Sodium bicarbonate group recorded the highest surface roughness mean values followed by hydrogen peroxide group then whitening paste group while control group recorded the lowest surface roughness mean value. The difference between whitening agent groups and control group was statistically significant $(\mathrm{p}<0.05)$ as indicated by two way ANOVA while the difference between whitening agent groups was statistically nonsignificant $(p>0.05)$ as indicated by Tukey's post hoc test presented in figure (1).

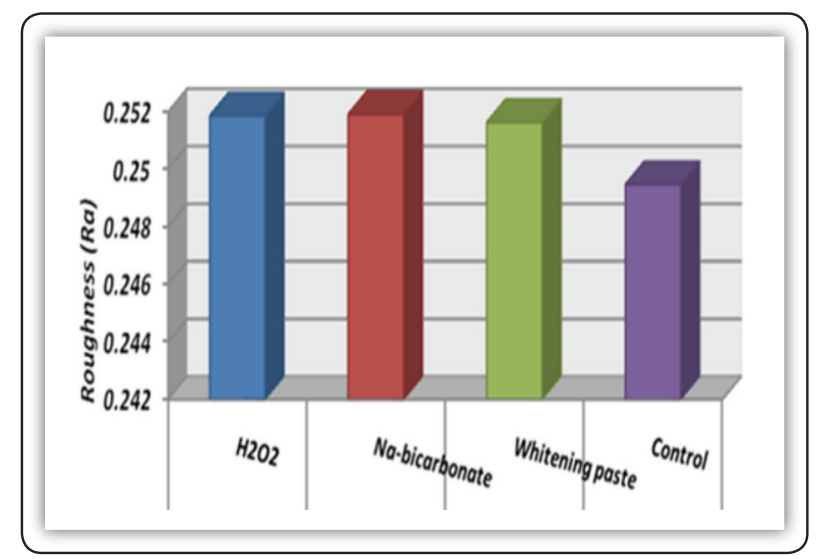

FIG (1) A column chart of total roughness mean values as a function of whitening agents groups.

\section{Effect of storage time on surface roughness}

The one day groups recorded the highest roughness mean values followed by the baseline then three months groups while six months groups recorded the lowest roughness mean value. The difference between different storage times and baseline was statistically significant $(\mathrm{p}<0.05)$ also the difference between different storage times was statistically significant $(\mathrm{p}<0.05)$ as indicated by two way ANOVA followed by Tukey's post hoc tests presented in figure (2).

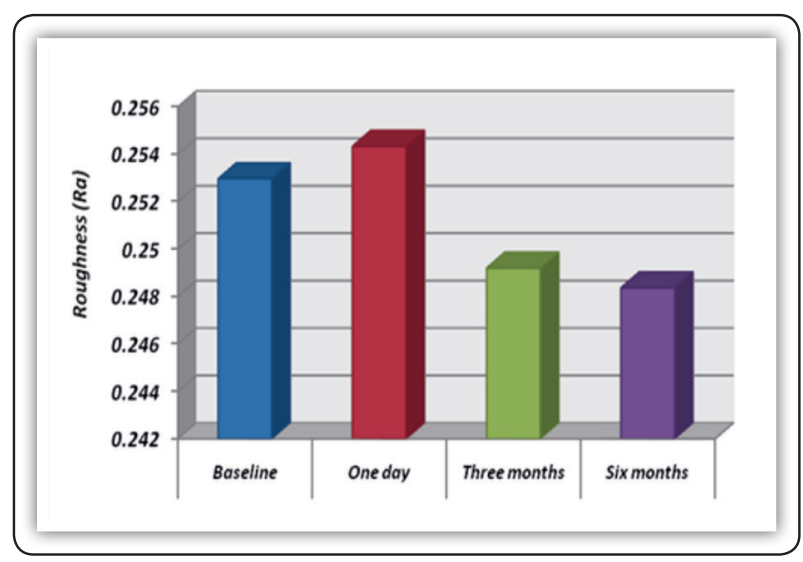

FIG (2) A column chart of total roughness mean values as a function of storage time.

\section{DISCUSSION}

Regarding the effect of home care whitening agents' application on surface roughness, the sodium bicarbonate powder brushing revealed the highest surface roughness; this may be related to the large abrasive particles of it. These abrasives are harder than the resin matrix and could even be similar in hardness to the fillers of the composite materials. So resin matrix is selectively removed, filler particles are exposed, resulting in a rough surface ${ }^{(11)}$.

The hydrogen peroxide revealed high surface roughness which may be related to its acidity, cause changes in the organic composition of resin composite, damages the polymer linkages and cause degradation ${ }^{(12)}$. Hydrogen peroxide demonstrates ability for diffusion and may result in a softening of the composite creating more interfaces which can affect filler degradation ${ }^{(13)}$. 
The whitening toothpaste brushing caused surface roughness because it contains abrasive particles as silica. The brushing with toothpaste might affect surface texture due to the retention of the abrasive agent in the composite surface ${ }^{(14)}$. The roughness which caused by whitening toothpaste brushing was lesser than sodium bicarbonate due to the abrasive particles of whitening toothpaste which is smaller than that of sodium bicarbonate particles ${ }^{(15)}$.

The control group received brushing with distilled water only. It showed the least surface roughness because the brushing without abrasives or chemicals causes less change in roughness ${ }^{(16)}$. Tooth brushing can erode the softer polymer matrix, leaving the harder reinforcing particles standing higher $^{(17)}$.

Regarding the effect of storage time, the highest surface roughness after one day may be due to the absorption of water which occurs as maximum in the first days ${ }^{(18)}$. The water causes deterioration of the resin matrix due to diffusion through it ${ }^{(19)}$. Water diffusion through the polymer chains and filler boundaries resulted in elution of components and the plasticization of the composite ${ }^{(20)}$. Also, there are changes such as volume expansion, softening and chemical changes as hydrolysis (21). Also, oxygen inhibited layer which is created against the celluloid matrix, rich in resinous monomers, less resistant to abrasion, have empty gaps and has unreacted monomers so can be diluted and washed away from the polymer matrix by the water ${ }^{(22)}$.

After 3 months and 6 months measurements, the surface roughness decrease, this may be due to silorane composition which has small hard filler particles situated as close as possible in order to protect the resin matrix from environment. Silorane composite contains quartz fillers which are more resistant to aqueous attack. Also, the monomer type directly influences the potential water sorption of the material. The resin of silorane has siloxane which increases the hydrophobicity ${ }^{(18)}$. Also all the components of the composite can leach out of the polymerized material so leaching may cause wear but the surface roughness may be decreased ${ }^{(23)}$.

\section{CONCLUSION}

1. Home care whitening agents had a significant effect on the surface roughness of low shrinkage composite.

2. Effect of home care whitening agents on the surface roughness was material dependent.

3. Storage in distilled water had an influential effect on the surface roughness of low shrinkage composite.

\section{REFERENCES}

1. Matis B, Cochran M and Eckert G. Review of the effectiveness of various tooth whitening systems. Oper Dent.2009; 34: 230-235.

2. Hasson H, Ismail A and Neiva G. Home-based chemically-induced whitening of teeth in adults. Cochrane Data Sys Rev.2006; 4: 1-43.

3. Teixeira E, Thompson J, Piascik J and Thompson JY. In vitro toothbrush-dentifrice abrasion of two restorative composites. J Esth Rest Dent.2005; 17: 172-180.

4. Imfeld T. Dental erosion, definition, classification and links. Eur J Oral Sci.1996; 104: 151-155.

5. Göhring T, Besek M and Schmidlin P. Attritional wear and abrasive surface alterations of composite resin materials in vitro. J Dent.2002; 30: 119-27.

6. Cannon M, Marshall G, Marshall S and Cooley R. Surface resistance to abrasion of preformed laminate resin veneers. J Prosth Dent.1984; 52: 323-330.

7. Nadhum $\mathrm{N}$ and Al-Khafaji A. The effect of in office bleaching on surface roughness and micro-hardness of newly developed composite materials (In vitro study) Bagh Coll Dent J.2014; 26: 24-29.

8. Grizon F, Mabilleau G and Chappard D. Abrasion of 6 dentifrices measured by vertical scanning interference microscopy. J Appli Oral Sci.2013; 21: 475-481.

9. Horcas I, Fernandez R, Gomez M, Colchero J, Gómez J and Baro A. Review of scientific instruments.2007; 78 013705 . 
10. Kakaboura A, Fragouli M and Rahiotis C. Evaluation of surface characteristics of dental composites using profilometry, scanning electron, atomic force microscopy and gloss-meter. J Mater Sci: Materials in Medicine.2007; 18: 155-163.

11. Roulet $\mathbf{J}$ and Roulet $\mathrm{T}$. The surface roughness of restorative materials and dental tissues after polishing with prophylaxis and polishing pastes. J Periodontol.1982; 53: 257-266.

12. Lima A, De Alexandre S, Martins C, Aguiar F, Ambrosano $\mathrm{G}$ and Lovadino J. Effect of curing light and bleching agents on physical properties of a hybrid composite resin. J Esthet Rest Dent.2008; 20: 266-275.

13. Attin T, Hannig C, Wiegand A and Attin R. Effect of bleaching on restorative materials and restorations- a systematic review. Dent Mater.2004; 20: 852-861.

14. Cury J, Rosing $\mathrm{C}$ and Tenuta L. Are dentifrices all the same?. Int J Braz Dent.2010; 6: 254-256.

15. Amaral M, Rodrigues A, Erhardt C, Araujo M, Marchi $\mathrm{G}$ and Pimenta L. Effect of whitening dentifrices on the superficial roughness of esthetic restorative materials. J Esthet Rest Dent.2006; 18: 102-108.

16. Tanoue N, Matsumura H and Atsuta M. Analysis of composite type and different sources of polymerization light on in vitro toothbrush/dentifrice abrasion resistance. J Dent. 2000; 28: 355-359.
17. Neme A, Frazier K, Roeder L and Debner T. Effect of prophylactic polishing protocols on the surface roughness of esthetic restorative materials.Oper Dent.2002; 27: 50-58.

18. Baydaa Hussein. Effect of casein phosphopeptide-amorphous calcium phosphate on surface roughness of a silorane-based and methacrylate-based composite resin (In vitro comparative study). Baghdad College Dentistry.2012; 24 : 106-112.

19. Sarret DC, Söderholm JM and Batich CD. Water and abrasive effects on three body wear of composites. Journal of Dental Research.1991; 70: 1074-1081.

20. Hahnel S, Henrich A, Bürgers R, Handel G and Rosentritt $M$. Investigation of mechanical properties of modern dental composites after artificial aging for one year. Operative Dentistry.2010; 35: 412-419.

21. Ferracane JL. Hygroscopic and hydrolytic effects in dental polymer networks. Dental Materials.2006; 22: 211-222.

22. Essam S. Shawkat, Adrian C. Shortall, Owen Addison and William M. Palin. Oxygen inhibition and incremental layer bond strengths of resin composites. Dental Materials.2009; 25: 1338-1346.

23. Geurtsen W. Substances released from dental resin composites and glass ionomer cements. European Journal of Oral Science.1998; 106: 687-695. 
